\title{
An Unusual Presentation of an Endometriotic Chocolate Cyst
}

1'Gaurav S Desai, ${ }^{2}$ Shyam V Desai

\section{ABSTRACT}

Background: Pelvic inflammatory disease is usually associated with gonococcal sexually transmitted infection. Its association with endometriosis has not been well documented.

Case report: The authors report an unusual case of an endometriotic cyst in a 29-year-old nulliparous patient with clinical features of pelvic inflammatory disease. On treatment with antibiotics, the symptomatology subsided and signs of infection diminished. But subsequent imaging revealed a persistent right adnexal mass. A right chocolate cyst was found on laparoscopy and was treated with drainage and enucleation. Patient was administered three doses of $\mathrm{GnRH}$ analogs thereafter and is awaiting treatment for infertility.

Conclusion: Endometriotic cysts commonly manifest with dyspareunia and dysmenorrhea. The patient in this report presented with signs and symptoms suggestive of pelvic inflammatory disease which was secondary to a slow leak from a chocolate cyst.

Keywords: Endometriosis pelvic inflammatory disease, Ovarian cyst, Chocolate cyst.

How to cite this article: Desai GS, Desai SV. An Unusual Presentation of an Endometriotic Chocolate Cyst. J South Asian Feder Obst Gynae 2014;6(1):46-48.

Source of support: Nil

Conflict of interest: None

\section{INTRODUCTION}

Endometriosis is a gynecological entity in which cells from the lining of the uterus (endometrium) appear and flourish outside the uterine cavity, most commonly on the membrane lining the abdominal cavity and the peritoneum. It is common in women, having an overall prevalence of 3 to $10 \%$ in the reproductive age group and about 25 to $35 \%$ in infertile women, and is responsible for a number of hospital admissions annually. ${ }^{1}$ Its origin, however, remains ambiguous if not unknown. Pelvic inflammatory disease (PID), on the other hand, encompasses a spectrum of symptoms and signs and may be acute, chronic, subacute or recurrent in its onset.

${ }^{1}$ Resident Physician, ${ }^{2}$ Professor and Honorary Consultant

1,2Department of Obstetrics and Gynecology, PD Hinduja Hospital and Research Center, Mumbai, Maharashtra, India

Corresponding Author: Gaurav S Desai, Resident Physician Department of Obstetrics and Gynecology, Hinduja Healthcare Surgical, Khar (W), Mumbai, Maharashtra, India, Phone: 912226482418, e-mail: gdesai83@gmail.com
It is usually associated with sexually transmitted infection such as gonococci and is responsible for a number of complications including tubal pregnancy from pelvic adhesions, infertility and pelvic masses. ${ }^{2}$ However, the manifestation of endometriosis as a pelvic infection is distinctly uncommon, and the paucity of reports in literature attests to this. ${ }^{3-6}$ This very relationship could potentially explain an additional presentation of an endometriotic cyst, thus adding another dimension to the complexity of this disorder. We report a case in which a patient with underlying endometriotic disease of the pelvis manifested with signs and symptomatology of pelvic inflammatory disease, and her subsequent treatment with antibiotics and laparoscopy.

\section{CASE REPORT}

A 29-year-old woman, nullipara, married since 18 months, came to the outpatient department on 29/6/13 with the complaint of lower abdominal pain, followed by low grade fever, two bouts of vomiting and an episode of diarrhea, all within the last 3 days. There was no history of surgical disorders or medical conditions requiring hospitalization, allergies or a family history which could be attributed to her presenting complaints. Her last menstrual period was on 19/6/13 and cycles were regular with heavy flow and dysmenorrhea.

Evaluation of her vital statistics revealed a pulse of 110/ min, a blood pressure of $100 / 60 \mathrm{~mm} \mathrm{Hg}$ and a temperature of $99.8^{\circ} \mathrm{F}$. Per abdominal examination showed tenderness in her lower abdomen, without signs of bowel perforation. There was thick discharge per speculum, and fullness in the pouch of Douglas on per vaginal examination, alongwith cervical motion tenderness. Laboratory investigations sent were as follows: blood group $\mathrm{B}$ positive, a $\mathrm{Hb}$ percentage of 10.5 and a TLC of $14,100 / \mathrm{mm}^{3}$. Her renal and liver function, coagulation profile and serum electrolytes were all within normal limits. Urine testing showed 150 to 160 pus cells/ hpf. However, a high vaginal swab and urine culture and sensitivity did not grow any organism.

Sonography was performed as per our institutional protocol and revealed a right sided adnexal mass suggestive of a pyosalpinx or hydrosalpinx. In an effort to further evaluate the cystic lesion, a CT scan was done which showed a right multiloculated thin smooth walled cystic lesion, measuring $6.5 \times 5 \times 5 \mathrm{~cm}$, with minimal free fluid in the POD and mild peritoneal enhancement, internal hemorrhage associated peritonitis. 
Conservative management was with intravenous antibiotics, i.e. injection piptaz (piperacillin and tazobactum) 4.5 gm 8 hourly, Inj. Amikacin (500 mg) 12 hourly, Inj. metronidazole ( $100 \mathrm{ml}$ ) IV 8 hourly was given for 5 days. In 24 hours, the patient became afebrile and in 48 hours she became asymptomatic and the WBC count reduced from $17500 / \mathrm{cmm}$ to $6500 / \mathrm{cmm}$. Thereafter a CT scan was done which showed persistant right cystic adnexal lesions, the lesion having mildly hyperdense contents. The right ovary was not seen separately. Both fallopian tubes had thick enhancing walls. There was minimal free fluid in the pelvis and in the right lower flank with enhancement of the adjacent peritoneum suggesting an infective or inflammatory etiology. The possibility of an endometriotic cyst could not be ruled out at this point in time. Also noted was mild appendiceal and cecal wall thickening, bilateral pleural effusion, minimal thickening of the gallbladder and pericholecystic edema.

An ultrasound repeated thereafter showed a normal sized uterus measuring $8.8 \times 5.3 \times 3.4 \mathrm{~cm}$, with an endometrial thickness of $6 \mathrm{~mm}$, a large well defined $5.8 \times 4.7 \mathrm{~cm}$ oval predominantly cystic mass in the right adnexa, with a well defined thick wall showing tightly packed coarse internal echoes. The left ovary was normal in size. There was minimal free fluid in the POD and right adnexal regions with coarse internal echoes within.

In view of the increased severity of symptoms and the findings on repeated imaging, a decision was taken for operative laparoscopy. Laparoscopy (Storz $10 \mathrm{~mm} 30^{\circ}$ laparoscope) was done under general anesthesia on 5/7/13. Intraoperatively, the omentum covered the uterus, with flimsy adhesions over both tubes and ovaries. Tubes, uterus and ovaries appeared hyperemic. A tubo-ovarian mass was seen in the right ovary. The left ovary and tube appeared healthy. Operative adhesiolysis and drainage of the endometriotic cyst in right ovary with cyst wall removal was done. Fluid from the POD was sent for culture and sensitivity, and that from the cyst wall for histopathology. Histology of the cyst wall showed an endometriotic cyst. POD fluid had an adenosine deaminase level of 36.7 and culture grew no organism. The post operative period was uneventful. The patient was discharged the coming evening. She has been given three doses of $\mathrm{GnRH}$ analogs thereafter and is awaiting treatment for infertility.

\section{DISCUSSION}

Possible complications of endometriosis include dysmenorrhea, disorders of menstruation, dyspareunia, dyschezia and infertility. Rarely, an endometriotic lesion can present with fatigue, diarrhea, constipation and bloating or nausea, especially during menstrual periods. However, pelvic infection has not been a well documented accompanying pathology of endometriosis. Our patient presented with PID and it was the persistance of adnexal lesions after conservative treatment with antibiotics that led us to the diagnosis of endometriotic cysts.

Abdominal pain in the setting of endometriosis usually arises from cysts and adhesions among pelvic organs. It is usually described as dull aching and can rarely be referred to the thigh or the back. A classic feature of the pain is that it usually undergoes cyclical exacerbations with each menstrual phase. Acute pain, on the other hand, can occur when a cyst ruptures. It should however be dissociated from other causes of acute abdomen including acute appendicitis or diverticulitis. Torsion of the endometriotic cyst could have possibly caused abdominal pain in our patient. However, this was not the case as we performed a color Doppler ultrasound at her initial presentation which showed sufficient blood flow to both ovaries. Nonetheless, our patient complained of an acute abdomen which was due to pelvic infection. In treating the PID with higher antibiotics, such as amikacin and piperacillin, the acute phase of the pathology, was arrested. However, the drainage and enucleation of the chocolate cyst was essential to correct any residual pathology due to endometriosis.

Reports in literature are few and far between the relationship of these two diseases. During a 6-year period, Moini et al studied 5958 transvaginal ultrasound-guided oocyte retrievals which resulted in 10 cases of acute PID $(0.12 \%)$. Eight of these 10 patients had endometriosis as a cause of infertility, one of whom had an endometrioma. They reported an increased risk of PID after oocyte retrieval in patients with endometriosis. ${ }^{3}$ As a fallout they recommended vigorous antibiotic prophylaxis and better vaginal preparation when oocyte pickup is performed in these patients. Gramatikakis et al studied the prevalence of PID in 720 women over 7-year period who underwent surgery due to endometriotic ovarian cysts and found it to be sufficiently higher than the prevalence in the general population. Laparoscopy was the treatment of choice in all the women with the exception of five cases, where due to technical difficulties during laparoscopy, a laparotomy was performed. In all the cases with PID, abscesses were evacuated laparoscopically. ${ }^{4}$ Van Weering et al reported a case of pelvic inflammatory disease and an abscessed endometriosis cyst in three women. ${ }^{5}$ Seaman et al investigated if the increased chance of having a diagnosis of irritable bowel syndrome (IBS) and pelvic inflammatory disease (PID) in women with endometriosis was due to misdiagnosis or comorbidity and stated that women with endometriosis were more likely to be diagnosed with irritable bowel syndrome and PID than controls, even after a definitive diagnosis of endometriosis or ovarian cysts. ${ }^{6}$ It may be confused with irritable bowel syndrome (IBS), a 
condition that causes bouts of diarrhea, constipation and abdominal cramping. IBS can accompany endometriosis, which can complicate the diagnosis.

It is very rare to have a patient presenting primarily with symptoms and signs of PID as in our case. Spontaneous rupture of an endometrioma or slow leakage can lead to an acute abdomen. As the intracystic contents of an endometrioma increase due to progressive growth, there is a possibility of a rupture of the endometrioma. At times trauma, as occurs during intercourse, may cause a rupture of the cyst leading to spillage of the contents into the peritoneal cavity and acute abdominal pain and emergency surgery. An acute inflammatory reaction which promotes dissemination of the disease and further adhesion formation may result. ${ }^{7}$

In the management of this case, the presence of endometriosis was overlooked due to the severely acute symptoms of PID and only when the acute episode of PID was controlled was the presence of the endometriotic cyst taken cognizance of. The diagnostic and operative laparoscopy was curative and following hospitalization the recovery of the patient was quick. She was discharged on the third day following surgery and a week after was back to work. A subsequent USG revealed no abnormality of the tubes and ovaries. The patient is now keen on a conception and is being worked upon to help her achieve a pregnancy.

\section{CONCLUSION}

The authors demonstrate the presence of chocolate cysts manifesting with clinical features suggestive of pelvic infection. The patient in this report presented with signs and symptoms suggestive of PID which was secondary to a slow leak from a chocolate cyst. The possibility of an endometriotic cyst should be considered in the presence of persistance adnexal masses after conservative antibiotic management. Its diagnosis is of utmost importance as negligence of the underlying pathology, i.e. endometriosis could lead to recurrence of pelvic infection and more importantly complications secondary to endometriosis.

\section{REFERENCES}

1. Daftary SN, Patki A. Reproductive endocrinology and infertility. BI Publication 2009.

2. Desai SV, Daftary SN. Endometriosis selected topics in obstetrics and gynaecology. For Postgraduates and Practitioners. BI Publications 2007;3.

3. Moini A, Riazi K, Amid V, et al. Endometriosis may contribute to oocyte retrieval-induced pelvic inflammatory disease: report of eight cases. J Assist Reprod Genet 2005;22(7-8):307-309.

4. Grammatikakis I, Evangelinakis N, Salamalekis G, et al. Prevalence of severe pelvic inflammatory disease and endometriotic ovarian cysts: a 7-year retrospective study. Clin Exp Obstet Gynecol 2009;36(4):235-236.

5. van Weering HG, Mijatovic V, Groot J, et al. Pelvic inflammatory disease and an abscessed endometriosis cyst: a diagnostic problem and a therapeutic dilemma. Ned Tijdschr Geneeskd. 2007;151(13):725-729.

6. Seaman HE, Ballard KD, Wright JT, de Vries CS. Endometriosis and its coexistence with irritable bowel syndrome and pelvic inflammatory disease: findings from a national case-control study Part 2. BJOG 2008;115(11):1392-1396.

7. Pratt JH, Shamblin WR. Spontaneous rupture of endometriotic cysts of the ovary presenting as an acute abdominal emergency Am J Obstet Gynaecol 1970;108:156-162. 